\title{
Chemotherapy-Induced Nausea and Vomiting (CINV) with GI Cancer Chemotherapy: Do We Need CINV Risk Score Over and Above Antiemetic Guidelines in Prescribing Antiemetic Regime?
}

\author{
Anita D'Souza1 Dipalee Pawar ${ }^{1}$ Anant Ramaswamy² Siddharth Turkar² \\ Prabhat Bhargava $^{2}$ Akhil Kapoor ${ }^{2}$ Sarika Mandavkar ${ }^{1} \quad$ Chaitali Nashikkar $^{2}$ Vikas Ostwal ${ }^{2}$
}

${ }^{1}$ Department of Nursing, GI Unit, Tata Memorial Hospital, Mumbai, Maharashtra, India

${ }^{2}$ Department of Medical Oncology, Tata Memorial Hospital Mumbai, Maharashtra, India
Address for correspondence Vikas Ostwal, DM, Department of Medical Oncology, Tata Memorial Hospital, Mumbai 400012 , Maharashtra, India (e-mail: dr.vikas.ostwal@gmail.com).

\section{South Asian J Cancer 2021;9:240-244.}

\section{Abstract}

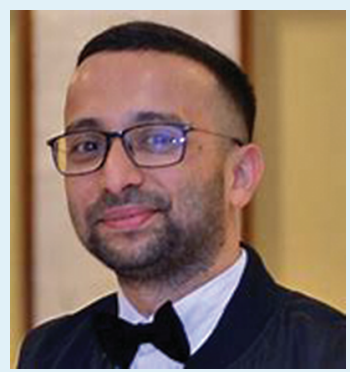

Dr Vikas Ostwal
Keywords

- CINV

- Gl cancer

- antiemetic

- chemotherapy

- assessment tool

- antiemetic guidelines
Background Various predictive models have been developed which incorporates patient risk factors into the selection of optimal antiemetic therapy, one of which is chemotherapy-induced nausea and vomiting (CINV) risk scoring system developed by Multinational Association of Supportive Care in Cancer (MASCC).

Patients and Methods Consecutive patients with gastrointestinal malignancy who had not received previous chemotherapy were eligible for enrollment in the study if they were scheduled to receive at least one cycle of chemotherapy. The CINV risk assessment tool was used to collect the study data and to assess CINV risk score.

Results Ninety-eight patients fulfilling the eligibility criteria were included in this study, out of which $57 \%$ were males, median age was 48 years (range: $28-77$ ). Colorectal cancer $(32.7 \%$ ) was the most common diagnosis followed by gastric cancer (27.6\%). Gemcitabine/cisplatin and CAPOX regimen were the most common regimen being administered in $19.4 \%$ each. As per MASCC guidelines, $19.4 \%$ patients received highly emetogenic chemotherapy, $69.4 \%$ moderately emetogenic chemotherapy, while $11.2 \%$ received regimen with low emetogenicity. CINV risk module characterized $52 \%$ patients to have high risk for CINV, while $48 \%$ to have low risk of CINV, thus, 52\% had the discrepancy in risk assigned by two methods, and this was statistically significant $(p=0.025)$. In subgroup analysis, although patient cohort with acute nausea had no statistically significant discrepancy $(p=0.123)$, but statistically significant discrepancy was found in patient cohort with delayed nausea $(p=0.001)$, acute $(p=0.038)$, and delayed $(p<0.001)$ vomiting.

Conclusion A significant percentage of patients who receive chemotherapy continue to experience nausea and vomiting despite receiving antiemetic treatment as per standard guidelines. The study generates a hypothesis for future large randomized studies looking at change in antiemetic prophylaxis based on CINV risk tool, leading to improvement in complete response rates of acute and delayed CINV.
DOI https://doi.org/10.1055/s-0041-1726136 ISSN 2278-330X.

How to cite this article: D'Souza A, Pawar D, Ramaswamy A Turkar S, Bhargava P, Kapoor A, Mandavkar S, Nashikkar C, Ostwal V. Chemotherapy-Induced Nausea and Vomiting (CINV) with GI Cancer Chemotherapy: Do We Need CINV Risk Score Over and Above Antiemetic Guidelines in Prescribing Antiemetic Regime?. South Asian J Cancer 2021;9(4):240-244.
(C) 2021. Medintel Services Pvt Ltd.

This is an open access article published by Thieme under the terms of the Creative Commons Attribution-NonDerivative-NonCommercial-License, permitting copying and reproduction so long as the original work is given appropriate credit. Contents may not be used for commercial purposes, or adapted, remixed, transformed or built upon. (https://creativecommons.org/licenses/by-nc-nd/4.0/).

Thieme Medical and Scientific Publishers Private Ltd A-12, Second Floor, Sector -2, NOIDA -201301, India 


\section{Introduction}

Chemotherapy-induced nausea and vomiting (CINV) is a bothersome side effect associated with cancer chemotherapy which adversely impacts both quality of life and the ability to carry out the activities of daily living. ${ }^{1-3}$ Moreover, patients with emesis may require emergency care or hospitalization which adds to the financial burden of cancer care. ${ }^{4-6}$ Moreover, patients with CINV become discouraged from completing their chemotherapy schedule.

Advances in our understanding of the pathophysiology of emesis and emergence of therapies to directly target these pathways have led to a remarkable improvement. Several guidelines have been published for preventing CINV in patients receiving cancer chemotherapy. ${ }^{78}$ However, these guidelines suffer major drawback in terms of not including individual patient factors that are well known to contribute to the emesis risk.

There is scarcity of data on incidence of CINV despite adherence to antiemetic guidelines. Few observational studies have reported around 15 to $70 \%$ incidence of CINV and these studies were done mostly on patients receiving highly emetogenic chemotherapy (HEC). ${ }^{5,6}$ However, very few studies have reported data with moderately emetogenic chemotherapy (MEC) which encompasses most of the gastrointestinal (GI) cancer chemotherapy regimens. Various predictive models have been developed which incorporates patient risk factors into the selection of optimal antiemetic therapy, one of which is CINV risk scoring system developed by Multinational Association of Supportive Care in Cancer (MASCC). ${ }^{9-11}$

The primary objectives of the current trial were to assess the proportion of patients developing CINV after receiving chemotherapy for GI cancers, in spite of receiving antiemetic prophylaxis as per the guidelines, as well as to assess the discrepancy in antiemetic therapy advised by guidelines vis-à-vis by CINV risk module. Secondary objective was to find out the proportion of patients developing CINV after receiving antiemetic treatment as per guidelines, who have discrepancy when assessed with respect to CINV risk module.

\section{Patients and Methods}

\section{Eligibility Criteria}

Patients 28 years of age or older with GI malignancy who had not received previous chemotherapy were eligible for enrollment in the study if they were scheduled to receive at least one cycle of chemotherapy for GI malignancy and had a Eastern Cooperative Oncology Group performance status of 0 to 2 (on a five-point scale, with 0 indicating no symptoms and higher numbers indicating increasing disability). Additional eligibility criteria were no clinical evidence of current or impending bowel obstruction or symptomatic brain metastases and no psychiatric/cognitive dysfunction interfering the compliance to antiemetic therapy.

\section{Study Design and Oversight}

In this study, prospective nonexperimental quasi purposive research design was used. The patients receiving chemotherapy for GI cancer (stomach, pancreas, gallbladder, and colorectal cancers) at Tata Memorial Hospital, Mumbai were included. The study was conducted in accordance with Good Clinical Practice, International Conference on Harmonization, and Declaration of Helsinki principles. Written informed consent was obtained from all patients. The study was approved by the Institutional Review Board (IEC/1119/3252/001). The authors vouch for the accuracy and completeness of the data and analysis and for adherence to the study protocol.

\section{Treatment Regimen}

All participants receiving HEC received a 5-HT3-receptor antagonist (palonosetron intravenously at a dose of $0.25 \mathrm{mg}$ or granisetron intravenously at a dose of $1 \mathrm{mg}$, with the specific agent chosen by the primary clinician) on day 1 of chemotherapy, dexamethasone (8-12 mg intravenously on day 1 , and $8 \mathrm{mg}$ orally on days 2-4), an neurokinin 1 (NK1)receptor antagonist (aprepitant $125 \mathrm{mg}$ orally on day 1 and $80 \mathrm{mg}$ on days 2 and 3 or fosaprepitant $150 \mathrm{mg}$ intravenously on day 1 ), and olanzapine (10 $\mathrm{mg}$ per day orally from days 1 to 4 ).

All participants receiving MEC received a two/three-drug combination of a 5-HT3-receptor antagonist and dexamethasone (8-12 mg intravenously on day 1 ) or a 5-HT3-receptor antagonist, a dexamethasone (8-12 mg intravenously on day 1), and an NK1-receptor antagonist. Adult patients who were treated with low-emetic-risk antineoplastic agents received either a single dose of a 5-HT3-receptor antagonist or a single 8-mg dose of dexamethasone before antineoplastic treatment. The doses of the antiemetics used in the study were as per the recommendations by various international guidelines for antiemetic agents.

\section{Study Visits and Assessment Procedures}

In the prestudy period, all pertinent demographic characteristics and clinical information on the cancer, metastases, chemotherapy regimen, administered prophylaxis for vomiting and/or nausea, and antiemetic medication prescribed were recorded by the investigator during the course of the study. The patients who agreed to participate in the study received a diary covering the first 5 days following chemotherapy administration. A study nurse contacted each patient at baseline and during the next chemotherapy visit to enquire about toxicities. Adverse events were graded according to the National Cancer Institute's Common Terminology Criteria for Adverse Events, version 5.0.

The CINV risk assessment tool was used to collect the study data and to assess CINV risk score. This tool is based on MASCC emetogenicity classification and considers chemotherapy factor as well as patient factor. It shows the category of emetogenicity (high, moderate, low, or minimal) of the chemotherapy drugs, percentage, and the patient risk factors such as gender, age, history of morning sickness in pregnancy, hours of sleep the night before chemotherapy, vomiting in prior cycle, and use of platinum-based chemotherapy. This tool generates the percentage of risk on the basis of combined factors and also classifies the risk as high 
or low. If both the modules categorize the same risk, then it was considered nondiscrepant. If the risk categories did not match, it was recorded as discrepancy.

The study was conducted over a period of 8 weeks and was started immediately after IEC approval. The purpose of the study was explained to the patient and willingness of the patient to participate in this study was ensured after which written informed consent was obtained.

\section{Statistical Analysis}

A descriptive analysis was performed on all of the variables (demographic variables and characteristics of the patients, the disease, and the chemotherapy received). For the primary objective, the incidence of CINV was evaluated during 5 days following administration. CINV within the first 24 hours following administration of the chemotherapy (acute phase) and during the 4 subsequent days (delayed phase) were recorded and expressed using the percentage of patients with nausea and vomiting. The CINV risk score was analyzed using percentage. The proportion and discrepancy between CINV and antiemetic guideline was analyzed using chi-square test and descriptive analysis. Logistic regression analysis was performed to explore the possibility of better classification of risk grouping based on the percentage risk of CINV calculated by the risk module. Statistical analysis was performed using Stata 14.2 (StataCorp). The comparative performances in predicting the risk of CINV were evaluated as (1) continuous variables derived from logistics regression, (2) CINV risk tool, and (3) guideline-based therapy. The cutoff for possible risk predictions were derived from logistic regression analysis.

\section{Results}

Around 300 patients who received chemotherapy for GI cancer were screened and 98 patients fulfilling the eligibility criteria were included in this study, out of which 56 patients (57\%) were males. Median age was 48 years (range: $28-77$ ). Most of the patients (80.6\%) were being treated with curative intent. Sixteen patients (16.3\%) had diabetes, $11.2 \%$ had hypertension, and $5.1 \%$ had both diabetes and hypertension.

Seventy-three patients (74.5\%) had a history of vomiting in previous cycle and 23 patients (23.5\%) slept for less than 7 hours the night prior to chemotherapy. Seventeen females (40.5\%) had a history of morning sickness during prior pregnancy (-Table 1 ).
Gemcitabine/cisplatin and CAPOX regimen were the most common regimen being administered in 19.4\% each, while $12.2 \%$ received FOLFIRI regimen. Other regimens included DOF (9.2\%), CAPE-IRI (7\%), DOX (6.1\%), FOLFIRINOX (6.1\%), FOLFOX (6.1\%), gemcitabine single agent (5.1\%), docetaxel single agent (3.1\%), and gemcitabine/nab paclitaxel (3.1\%) (-Supplementary Table S1, online only).

Incidence and grades of acute and delayed CINV are discussed in - Table 2 and - Supplementary Table S2 (online only).

Table 1 Baseline characteristics of participants

\begin{tabular}{|c|c|}
\hline Characteristic & $\begin{array}{l}\text { Number of patients (\%), } \\
n=98\end{array}$ \\
\hline \multicolumn{2}{|l|}{ Gender } \\
\hline Male & $56(57.1)$ \\
\hline Female & $42(42.8)$ \\
\hline \multicolumn{2}{|l|}{ Age group (y) } \\
\hline $28-38$ & $14(14.3)$ \\
\hline $39-49$ & $41(41.8)$ \\
\hline $50-60$ & $29(29.6)$ \\
\hline $61-70$ & $14(14.3)$ \\
\hline \multicolumn{2}{|l|}{ Site of primary } \\
\hline Colon & $21(21.4)$ \\
\hline Cholangiocarcinoma & $23(23.5)$ \\
\hline Pancreas & $14(14.3)$ \\
\hline Rectum & $13(13.3)$ \\
\hline Stomach & $27(27.6)$ \\
\hline \multicolumn{2}{|l|}{ Addictions } \\
\hline History of tobacco intake & $12(12.2)$ \\
\hline $\begin{array}{l}\text { History of alcohol } \\
\text { consumption }\end{array}$ & $6(6.1)$ \\
\hline \multicolumn{2}{|l|}{ Patient risk factors for CINV } \\
\hline $\begin{array}{l}\text { History of morning sick- } \\
\text { ness in prior pregnancy } \\
\text { (out of } 42 \text { female patients) }\end{array}$ & $17(40.47)$ \\
\hline $\begin{array}{l}\text { History of nausea or vom- } \\
\text { iting episode in previous } \\
\text { cycle }\end{array}$ & $73(74.5)$ \\
\hline $\begin{array}{l}\text { History of sleep }<7 \\
\text { h the night before } \\
\text { chemotherapy }\end{array}$ & $23(23.5)$ \\
\hline
\end{tabular}

Abbreviation: CINV, chemotherapy-induced nausea vomiting.

Table 2 Incidence of CINV events

\begin{tabular}{|l|l|l|l|l|}
\hline Grade & Acute nausea & Delayed nausea & Acute vomiting & Delayed vomiting \\
\hline Grade 0 & $65(66.3)$ & $28(28.6)$ & $80(81.6)$ & $62(63.3)$ \\
\hline Grade 1 & $21(21.4)$ & $26(26.5)$ & $7(7.1)$ & $11(11.2)$ \\
\hline Grade 2 & $8(8.2)$ & $32(32.7)$ & $8(8.2)$ & $20(20.4)$ \\
\hline Grade 3 & $4(4.1)$ & $12(12.2)$ & $3(3.1)$ & $4(4.1)$ \\
\hline Grade 4 & - & - & $0(0)$ & $1(1.0)$ \\
\hline
\end{tabular}

Abbreviation: CINV, chemotherapy-induced nausea vomiting. 
Table 3 CINV Risk prediction as per tool

\begin{tabular}{|l|l|l|l|l|}
\hline $\begin{array}{l}\text { Predicting complete } \\
\text { response }\end{array}$ & Acute CINV (AUC) & Delayed CINV (AUC) & \\
\hline $\begin{array}{l}\text { Continuous variables } \\
\text { assessment methods }\end{array}$ & $\begin{array}{l}(64.0 \%, 95 \% \mathrm{Cl}: \\
53.2-73.7)\end{array}$ & $\begin{array}{l}\text { vs. guidelines } \\
p=0.045 . \\
\text { vs. CINV module, } \\
p=0.590\end{array}$ & $\begin{array}{l}(76.1 \%, 95 \% \mathrm{Cl}: \\
66.7-85.5)\end{array}$ & $\begin{array}{l}\text { vs. guidelines } \\
p=0.025 \\
\text { vs. CINV module, } \\
p=0.590\end{array}$ \\
\hline CINV risk module & $\begin{array}{l}\text { vs. guidelines } \\
p=0.060\end{array}$ & $\begin{array}{l}(67.9 \%, 95 \% \mathrm{Cl}: \\
58.3-77.40)\end{array}$ & $\begin{array}{l}\text { vs. guidelines } \\
p=0.038\end{array}$ \\
\hline Guidelines & $\begin{array}{l}(58.1-70.1) \\
(51.8 \%, 95 \% \mathrm{Cl}:\end{array}$ & $58.6 \%, 95 \% \mathrm{Cl}: 48.3-68.9$ & \\
\hline
\end{tabular}

Abbreviations: AUC, area under the curve; $\mathrm{Cl}$, confidence interval; CINV, chemotherapy-induced nausea vomiting.

\section{Categorization Based on Chemotherapy Regime}

As per MASCC guidelines, 19.4\% patients received HEC, 69.4\% MEC, while $11.2 \%$ received regimen with low emetogenicity (- Table 3). CINV risk module characterized $52 \%$ patients to have high risk for CINV, while $48 \%$ to have low risk of CINV. Thus, $52 \%$ patients had the discrepancy in risk assigned by two methods, and this was statistically significant $(p=0.025)$.

\section{Discrepancy Based on Development of Acute and Delayed CINV}

Patient cohort with acute nausea had no statistically significant discrepancy $(p=0.123)$, but statistically significant discrepancy was found in patient cohort with delayed nausea $(p=0.001)$, acute $(p=0.038)$, and delayed $(p<0.001)$ vomiting.

\section{Prediction of CR of Acute and Delayed CINV}

- Supplementary Table S3 (online only) and - Table 3 explain area under the curve (AUC) and CINV risk tools that have better predictive values than guidelines.

Eighteen (26.4\%) patients who were classified as receiving MEC were found to have high risk of CINV by the risk module. Logistic regression analysis suggested the classification of percentage of CINV risk into four groups: $\leq 25,26$ to 35,36 to 45 , and $>45 \%$ (-Table 3 ).

\section{Discussion}

Current recommendations for CINV prophylaxis is based primarily on the emetogenic potential of the drugs selected for chemotherapy, which have been classified into four emetogenic risk groups: high, moderate, low, and minimal. However, the standard guidelines do not take individual factors into association leading to possible failure to predict accurately the risk of CINV. There is a huge unmet need.

We found that almost $45 \%$ of patients had more than or equal to grade 2 nausea in spite of antiemetic prophylaxis use as per guidelines. This is comparable to the study by Dranitsaris et al which reported that $42.2 \%$ of patients experienced more than grade 2 CINV despite appropriate antiemetics as per standard guidelines. ${ }^{9}$ Hernandez Torres et al also reported nausea in $71 \%$ patients and vomiting in $26 \%$ patients which is parallel to our results. ${ }^{12}$ Study done by Baburaj et al also showed that the incidence of CINV was 69.5 and $46 \%$, respectively, ${ }^{13}$ while Zong et al reported the incidence to be $65.38 \%$ and vomiting $50 \%$, respectively. ${ }^{14}$

Delayed CINV continues to be an unmet clinical need and its incidence in this study was considerably high (71.4 and $36.7 \%$, respectively) which is comparable to the study done by Escobar et al in patients receiving MEC in colorectal cancer. ${ }^{15}$

In our study, more than $25 \%$ patients who were classified as receiving MEC (based on guidelines) were found to have high risk of CINV by the risk module, and additional antiemetics were suggested for these patients by the risk module. Thus, the use of risk module (incorporation of patient factors besides chemotherapy emetogenicity risk) might be an important strategy for further improving the complete response (CR) rates for CINV.

Multiple previous studies have highlighted the impact of CINV on extra hospital visits, cost of treatment, and need of emergency services. ${ }^{4-6,16}$ There is still substantial room for improvement in reducing CINV health care resource utilization following HEC or MEC. The clinical application of this CINV risk prediction tool can serve as an important tool to assess patient-specific CINV risk and may optimize the use of the antiemetics in a proactive manner.

The primary limitations of this study consist of the consequences of its observational design and the lack of follow-up until several cycles had been completed. Also, there could be errors or omissions when patients made entry in the diary. Further studies are also needed to evaluate the impact of CINV on health care resource use especially in resource-limited setting like India. Besides, this study found the discrepancy in the prediction of emetogenicity risk but was unable to find that better prediction of CINV risk will actually result in improvement of $\mathrm{CR}$ rates. This will require a well-designed study with larger number of patients to answer this question.

In conclusion, a significant percentage of patients who receive chemotherapy continue to experience nausea and vomiting despite receiving antiemetic treatment as per standard guidelines. The study generates a hypothesis for future large randomized studies looking at change in antiemetic prophylaxis based on CINV risk tool, leading to improvement in CR rates of acute and delayed CINV. 
Source of Support

None.

Conflict of Interest

None declared.

\section{References}

1 Bloechl-Daum B, Deuson RR, Mavros P, Hansen M, Herrstedt J. Delayed nausea and vomiting continue to reduce patients' quality of life after highly and moderately emetogenic chemotherapy despite antiemetic treatment. J Clin Oncol 2006;24(27):4472-4478

2 Cohen L, de Moor CA, Eisenberg P, Ming EE, Hu $\mathrm{H}$. Chemotherapy-induced nausea and vomiting: incidence and impact on patient quality of life at community oncology settings. Support Care Cancer 2007;15(5):497-503

3 Glaus A, Knipping C, Morant R, et al. Chemotherapy-induced nausea and vomiting in routine practice: a European perspective. Support Care Cancer 2004;12(10):708-715

4 Ihbe-Heffinger A, Ehlken B, Bernard R, et al. The impact of delayed chemotherapy-induced nausea and vomiting on patients, health resource utilization and costs in German cancer centers. Ann Oncol 2004;15(3):526-536

5 Ballatori E, Roila F, Ruggeri B, et al. The cost of chemotherapy-induced nausea and vomiting in Italy. Support Care Cancer 2007;15(1):31-38

6 Lachaine J, Yelle L, Kaizer L, Dufour A, Hopkins S, Deuson R. Chemotherapy-induced emesis: quality of life and economic impact in the context of current practice in Canada. Support Cancer Ther 2005;2(3):181-187

7 MASCC and ESMO Consensus Guidelines for the Prevention of Chemotherapyand Radiotherapy-Induced NauseaandVomiting. ESMO Clinical Practice Guidelines | ESMO [Internet]. (Cited July 19, 2019). Available at: https://www.esmo.org/Guidelines/ Supportive-and-Palliative-Care/MASCC-and-ESMO-Consensus-Guidelines-for-the-Prevention-of-Chemotherapy-andRadiotherapy-Induced-Nausea-and-Vomiting
8 Hesketh PJ, Kris MG, Basch E, et al. Antiemetics: American Society of Clinical Oncology clinical practice guideline update. J Clin Oncol 2017;35(28):3240-3261

9 Dranitsaris G, Molassiotis A, Clemons M, et al. The development of a prediction tool to identify cancer patients at high risk for chemotherapy-induced nausea and vomiting. Ann Oncol 2017;28(6):1260-1267

10 Dranitsaris G, Bouganim N, Milano C, et al. Prospective validation of a prediction tool for identifying patients at high risk for chemotherapy-induced nausea and vomiting. J Support Oncol 2013;11(1):14-21

11 Molassiotis A, Stamataki Z, Kontopantelis E. Development and preliminary validation of a risk prediction model for chemotherapy-related nausea and vomiting. Support Care Cancer 2013;21(10):2759-2767

12 Hernandez Torres C, Mazzarello S, Ng T, et al. Defining optimal control of chemotherapy-induced nausea and vomiting-based on patients' experience. Support Care Cancer 2015;23(11):3341-3359

13 Baburaj G, Abraham AM, George L, et al. A study on utilization and evaluation of antiemetics in chemotherapy-induced nausea and vomiting. Indian J Med Paediatr Oncol 2017;38(3):334-339

14 Zong X, Zhang J, Ji X, Gao J, Ji J. Patterns of antiemetic prophylaxis for chemotherapy-induced nausea and vomiting in China. Chin J Cancer Res 2016;28(2):168-179

15 Escobar Y, Cajaraville G, Virizuela JA, et al. Incidence of chemotherapy-induced nausea and vomiting with moderately emetogenic chemotherapy: ADVICE (Actual Data of Vomiting Incidence by Chemotherapy Evaluation) study. Support Care Cancer 2015;23(9):2833-2840

16 Burke TA, Wisniewski T, Ernst FR. Resource utilization and costs associated with chemotherapy-induced nausea and vomiting (CINV) following highly or moderately emetogenic chemotherapy administered in the US outpatient hospital setting. Support Care Cancer 2011;19(1):131-140

\section{Certificate Course in Telemedicine Practice Guidelines}

If you want to get certified in doing Telemedicine Consultation (as per requirements of Govt of India notification), please enrol for the six module online course at:

https://www.onference.in/c/certificate-course-in-telemedicine-by-acdh/1610 